\title{
SUMS OF SYMMETRICAL RANDOM VARIABLES
}

\author{
D. A. DARLING
}

1. Introduction. In this paper we deal with sums of random variables $X_{1}, X_{2}, \cdots$, which have the following properties: The $X_{i}$ are independent, identically distributed, and have a common continuous symmetrical distribution. A random variable $X$ is symmetrical if $\operatorname{Pr}\{X<a\}=\operatorname{Pr}\{X>-a\}$ for every $a$.

As is well known, even if the symmetry condition is waived, any statistic which depends only on the relative magnitude of the $X_{i}$ is distribution free; that is, its distribution is independent of the parent distribution of the $X_{i}$. Letting $S_{n}=X_{1}+{ }_{2} X+\cdots+X_{n}$, $S_{0}=0$, it turns out that there are certain order relations among the $S_{j}$ which are also distribution free if the $X_{i}$ have the properties mentioned above.

E. S. Andersen [1] ${ }^{1}$ has shown that if $N_{n}$ is the number of positive $S_{j}(j=1,2, \cdots, n)$ then $\operatorname{Pr}\left\{N_{n}=k\right\}$ is independent of the distribution of the $X_{i}$. The limiting case of this theorem when $n \rightarrow \infty$ was established earlier by Erdös and Kac [2] under somewhat different assumptions about the $X_{j}$. In the present paper these results, and others, are obtained by specializing a somewhat more general theorem whose proof is relatively simple.

The central proposition (Theorem 1), which is believed to include all the distribution free properties of the $S_{j}$, is the following: Since the distribution of the $X_{i}$ is continuous there are, with probability one, no equalities between any two of the $S_{j}$. Then the random variables $S_{0}, S_{1}, \cdots, S_{n}$, when put in ascending order, will induce a random permutation among the $n+1$ integers $0,1, \cdots, n$ to give $S_{I_{0}}<S_{I_{1}}<\cdots<S_{I_{n}}$. Then $I_{j}$ is a random variable taking on the values $0,1, \cdots, n$. If we put

$$
p_{i, k}(n)=\operatorname{Pr}\left\{I_{k}=j\right\}
$$

it turns out that $p_{j, k}(n)$ is an absolute number independent of the distribution of the $X_{i}$ (subject to the requirements cited in the opening paragraph).

From this theorem there follow several interesting consequences. We obtain the distribution of that value of $j$ for which $S_{j}$ attains its maximum, $j=0,1, \cdots, n$, and the distribution of the number of positive $S_{j}, j=1,2, \cdots, n$. For $n \rightarrow \infty$ there result corresponding

Presented to the Society, April 29, 1949; received by the editors August 21, 1950.

${ }^{1}$ Numbers in brackets refer to the bibliography at the end of the paper. 
limiting theorems which for a suitable distribution of the $X$ 's yield analogous properties of the Wiener stochastic process.

2. The principal theorem. In the sequel some of the remarks made will be true only with probability one, but for brevity this qualification will of ten not be explicitly stated. Let the distribution of the $X_{i}$ and the definition of the $S_{j}$ be as given in the preceding section, and we suppose that $\left(I_{0}, I_{1}, \cdots, I_{n}\right)$ is a permutation of $(0,1, \cdots, n)$ such that $S_{I_{0}}<S_{I_{1}}<\cdots<S_{I_{n}}$, and for abbreviation we denote the event that $I_{k}=j$ by $A_{j, k}(n)$ and $\operatorname{Pr}\left\{A_{j, k}(n)\right\}=p_{j, k}(n)$. The event $A_{j, k}(n)$ means simply that there are $k$ terms $S_{i}$ which are less than $S_{j}$ and $n-k$ greater than $S_{j}$ among the sequence $S_{0}=0, S_{1}, S_{2}, \cdots, S_{n}$.

We have the following theorem.

THEOREM 1.

$$
p_{j, k}(n)=\operatorname{Pr}\left\{A_{j, k}(n)\right\}=\sum_{\nu=\max (0, j+k-n)}^{\min (j, k)} u_{\nu} u_{k-\nu} u_{j-\nu} u_{n-k-j+\nu}
$$

where

$$
u_{i}=\frac{1}{2^{2 i}} C_{2 i, i}
$$

Proof. We make a simple enumeration of the mutually exclusive and exhaustive ways in which $I_{k}$ can equal $j$. We have $I_{k}=j$ if and only if for some value of $\nu$ the following event occurs: Among the $S_{\mu}$ for $\mu<j$ there are $\nu$ terms less than $S_{j}$ and simultaneously among the $S_{\mu}$ for $j<\mu \leqq n$ there are $k-\nu$ terms less than $S_{j}$. Clearly this event cannot happen for two different values of $\nu$, and $\nu$ is restricted by the relation $\max (0, j+k-n) \leqq \nu \leqq \min (j, k)$.

If we consider the set of random variables $-X_{j},-X_{j-1}, \cdots,-X_{1}$ and recall the fact that the $X_{i}$ are symmetrically distributed and we form from this sequence the corresponding set of partial sums $S_{0}^{\prime}=0, S_{1}^{\prime}, S_{2}^{\prime}, \cdots, S_{j}^{\prime}$, then the event $A_{r, s}^{\prime}(j)$ formed from these random variables will have the same probability as $A_{r, s}(j)$ formed from the original sequence $S_{0}, S_{1}, \cdots, S_{j}$. Now, with the event that there are $\nu$ terms among the $S_{0}, S_{1}, \cdots, S_{j-1}$ which are less than $S_{j}$ we have the identical event $A_{0, \nu}^{\prime}(j)$ by considering the sequence $S_{0}^{\prime}$, $S_{1}^{\prime}, \cdots, S_{j}^{\prime}$. Similarly by considering the set of variables $X_{j+1}$, $X_{j+2}, \cdots, X_{n}$ we obtain a set of partial sums $S_{0}^{\prime \prime}=0, S_{1}^{\prime \prime}, \cdots, S_{n-j}^{\prime \prime}$ and the event that $k-\nu$ of the $S_{\mu}$ for $j<\mu \leqq n$ are less than $S_{j}$ becomes the event $A_{0, k-\nu}^{\prime \prime}(n-j)$ in connection with the suite $S_{0}^{\prime \prime}$, $S_{1}^{\prime \prime}, \cdots, S_{n-j}^{\prime \prime}$, and again $\operatorname{Pr}\left\{A_{r, 8}^{\prime \prime}(n-j)\right\}=\operatorname{Pr}\left\{A_{r, 8}(n-j)\right\}$. Thus we finally obtain 


$$
A_{j, k}(n)=\sum_{\nu=\max (0, j+k-n)}^{\min (j, k)} A_{0, \nu}^{\prime}(j) \cap A_{0, k-\nu}^{\prime \prime}(n-j) .
$$

The terms in this sum are mutually exclusive events, and the two members in each intersection are independent events, for $A_{0, \nu}^{\prime}(j)$ depends only on those $X_{i}$ for $i \leqq j$ and $A_{0, k-\nu}^{\prime \prime}(n-j)$ depends only on those $X_{i}$ for $i>j$, and the $X_{i}$ are presumed to be independent. If we take probabilities of both sides of the above expression, and use the fact that the events $A, A^{\prime}$, and $A^{\prime \prime}$ are equiprobable, we obtain

$$
p_{j, k}(n)=\sum_{\nu=\max (0, j+k-n)}^{\min (j, k)} p_{0, \nu}(j) p_{0, k-\nu}(n-j) .
$$

Exactly one of the events

$$
A_{0, k}(n), A_{1, k}(n), \cdots, A_{n, k}(n)
$$

must occur (that is, $S_{j}$ is the $k$ th largest partial sum for exactly one value of $j$ ) so that summing this expression, we obtain

$$
\begin{aligned}
& 1=\sum_{j=0}^{n} \sum_{\nu=\max (0, j+k-n)}^{\min (j, k)} p_{0, \nu}(j) p_{0, k-\nu}(n-j), \\
& 1=\sum_{\nu=0}^{k} \sum_{j=\nu}^{n-k+\nu} p_{0, \nu}(j) p_{0, k-\nu}(n-j) .
\end{aligned}
$$

This formula enables us to find recursively the $p_{0, k}(n)$ for $k$ $=0,1, \cdots, n, n=0,1, \cdots$, after putting $p_{0,0}(0)=1$. Using these in (2) will enable us finally to establish Theorem 1.

To actually evaluate the $p_{j, k}(n)$ it appears simplest to use the method of generating functions. Let us put

$$
\phi_{\nu}(x)=\sum_{j=\nu}^{\infty} p_{0, \nu}(j) x^{i}
$$

and note that the inner sum of (3) is a simple convolution. Thus multiplying (3) through by $x^{n}$ and summing from $n=k$ to $\infty$ (which is clearly permissible if $|x|<1$ ), we obtain

$$
\frac{x^{k}}{1-x}=\sum_{\nu=0}^{k} \phi_{\nu}(x) \phi_{k-\nu}(x) \text {. }
$$

This is again in the form of a convolution, and if we let

$$
\psi(x, y)=\sum_{v=0}^{\infty} \phi_{\nu}(x) y^{\prime}
$$


we have, repeating the above process,

$$
\psi^{2}(x, y)=\frac{1}{(1-x)(1-x y)}
$$

so that $\psi(x, y)=((1-x)(1-x y))^{-1 / 2}$. If we let $u_{r}=\left(1 / 2^{2 r}\right) C_{2 r, r}$, then $(1-y)^{-1 / 2}=\sum_{r=0}^{\infty} u_{r} y^{r}$, so that

$$
\phi_{\nu}(x)=\frac{u_{\nu} x^{\nu}}{(1-x)^{1 / 2}}
$$

and finally, obtaining the coefficient of $x^{n}$ in a power series expansion of this expression, we obtain

$$
p_{0, \nu}(n)=u_{\nu} u_{n \rightarrow \nu}
$$

Upon substituting this in (2) we obtain Theorem 1.

3. Two corollaries. From Theorem 1 two interesting consequences follow as special cases. The first of these is the following corollary.

Corollary 1. Let $M_{n}$ be that value of $j$ for which $S_{j}$ attains its maximum for $j=0,1, \cdots, n$. Then

$$
\operatorname{Pr}\left\{M_{n}=k\right\}=p_{0, k}(n)=u_{k} u_{n-k} .
$$

We have $\operatorname{Pr}\left\{M_{n}=k\right\}=\operatorname{Pr}\left\{I_{n}=k\right\}=p_{k, n}(n)$ and from Theorem 1 it is clear that $p_{j, k}(n)=p_{k, j}(n)=p_{k, n-j}(n)$ for all $k$ and $j$. Hence $p_{k, n}(n)=p_{0, k}(n)$ and the assertion is proved. Naturally, a similar remark holds for the minimum.

We also obtain the following corollary.

CoRollary 2. Let $N_{n}$ be the number of positive $S_{j}$ for $j=1,2, \cdots, n$. Then $\operatorname{Pr}\left\{N_{n}=k\right\}=p_{0, k}(n)=u_{k} u_{n-k}$.

This result is immediately established by noting that if $I_{n-k}=0$, then, since $S_{0}=0$, exactly $n-k$ of the sums are negative and $k$ are positive. Since $p_{0, n-k}(n)=p_{0, k}(n)$, the corollary follows.

Corollary 2 has been proven in an entirely different way by E. S. Andersen, using combinatorial methods [1]. In the present work, done independently of Andersen's research, it should be remarked that to prove Corollary 2 directly without first obtaining Theorem 1 seems very difficult, using the methods of this paper.

4. The limiting cases. Using the notation

$$
\mathrm{sn}^{-1} k=\int_{0}^{1} \frac{d x}{\left(\left(1-x^{2}\right)\left(1-k^{2} x^{2}\right)\right)^{1 / 2}}, \quad k^{2}<1,
$$


we define the following function:

$$
f(\alpha, \beta)=\left\{\begin{array}{c}
\frac{2}{\pi^{2}(\alpha(1-\alpha))^{1 / 2}} \operatorname{sn}^{-1}\left(\left(\frac{\beta(1-\beta)}{\alpha(1-\alpha)}\right)^{1 / 2}\right), \\
\frac{2}{2} \sin ^{-1}\left(\left(\frac{\alpha(1-\alpha)}{\beta(1-\beta)}\right)^{1 / 2}\right), \\
\beta(1-\beta)>\alpha(1-\alpha),
\end{array}\right.
$$

and $f(\alpha, \beta)$ is defined everywhere in the unit square $0 \leqq \alpha \leqq 1,0 \leqq \beta \leqq 1$ except for the points for which $\alpha(1-\alpha)=\beta(1-\beta)$. These points lie on the lines $\alpha-\beta=0$ and $\alpha+\beta=1$, and near them $f(\alpha, \beta)$ becomes large.

We have the following limiting theorem.

THEOREM 2.

$$
\lim _{n \rightarrow \infty} \operatorname{Pr}\left\{I_{[n \alpha]}<n \beta\right\}=\int_{0}^{\beta} f(\alpha, \xi) d \xi, \quad \alpha \leqq 1, \beta \leqq 1 .
$$

The function $f(\alpha, \beta)$ is thus seen to be for every fixed $\alpha$ a density on $\beta$ and vice versa since $f(\alpha, \beta)=f(\beta, \alpha)$. To prove the theorem we suppose initially that $\alpha(1-\alpha) \neq \beta(1-\beta)$ and note that $u_{r}=2^{-2 r} C_{2 r, r}$ $\sim(\pi r)^{-1 / 2}, r \rightarrow \infty$. Then it is simple to verify that this asymptotic value can be used, in the limit for large $n$, in the sum

$$
p_{j, k}(n)=\sum_{\nu=\max (0, j+k-n)}^{\min (j, k)} u_{\nu} u_{j-\nu} u_{k-\nu} u_{n-j-k+\nu}
$$

to give

$$
\begin{aligned}
& p_{j, k}(n) \sim \frac{1}{\pi^{2} n^{2}} \sum_{\nu=\max (0, j+k-n)}^{\min (j, k)}\left(\frac{\nu}{n}\left(\frac{j}{n}-\frac{\nu}{n}\right)\left(\frac{k}{n}-\frac{\nu}{n}\right)\right. \\
& p_{[n \alpha],[n]\}}(n)\left.\left.\sim \frac{1}{\pi^{2} n} \int_{\max (0, \alpha+\beta-1)}^{\min (\alpha, \beta)} \frac{j}{n}-\frac{k}{n}+\frac{\nu}{n}\right)\right)^{-1 / 2}, \\
&=\frac{1}{n} f(\alpha, \beta)
\end{aligned}
$$

by a well known transformation of elliptic integrals. If we suppose that $\gamma$ and $\beta$ are such that $\xi(1-\xi) \neq \alpha(1-\alpha)$ for $\gamma \leqq \xi \leqq \beta$, then 


$$
\operatorname{Pr}\left\{n \gamma \leqq I_{[n \alpha]}<n \beta\right\}=\sum_{n \gamma \leqq \nu<n \beta} p_{\nu,[n \alpha]}(n) \sim \int_{\gamma}^{\beta} f(\alpha, \xi) d \xi .
$$

The exceptional values of $\alpha, \beta$, and $\gamma$ are now seen to contribute a negligible amount to the total probability, and the latter integral can be made a continuous function of $\alpha, \beta$, and $\gamma$ by a proper assignment at its undefined values. This done, the function $\int_{0}^{\beta} f(\alpha, \xi) d \xi$ is a continuous distribution function on $\beta$ for every $\alpha(0 \leqq \beta \leqq 1,0 \leqq \alpha \leqq 1)$, and Theorem 2 is proved.

If we put $\alpha$ (or $\beta$ ) equal to zero we obtain, corresponding to Corollaries 1 and 2, the following corollary.

Corollary 3. Let $R_{n}$ equal either $M_{n}$ (the value of $j$ for which $S_{j}$ attains its maximum, $j=0,1, \cdots, n$ ) or $N_{n}$ (the number of positive $\left.S_{j}, j=1,2, \cdots, n\right)$. Then

$$
\lim _{n \rightarrow \infty} \operatorname{Pr}\left\{R_{n}<n \alpha\right\}=\frac{2}{\pi} \sin ^{-1} \alpha^{1 / 2} .
$$

This limiting expression is clearly $\int_{0}^{\alpha} f(0, \xi) d \xi=(1 / \pi) \int_{0}^{\alpha} d \xi /(\xi(1-\xi))^{1 / 2}$ $=(2 / \pi) \sin ^{-1} \alpha^{1 / 2}$ as asserted. For the variable $N_{n}$ this limiting expression was proven by Erdös and Kac [2] under somewhat different conditions on the distribution of the $X_{i}$.

5. Connection with the Wiener process. The limiting theorem given in the preceding section has an interpretation in terms of the Wiener stochastic process. If, for instance, $E\left(X_{k}^{2}\right)=\sigma^{2}<\infty$, then $X_{n}(t)=S_{[n t]} / \sigma n^{1 / 2}$ is a random variable which will, for large $n$, reflect the properties of the Wiener process.

Thus if $X(t)$ is an element of Wiener space, then the set of $t$ for which $X(t)<X(\alpha)(0 \leqq t \leqq 1,0 \leqq \alpha \leqq 1)$ is with probability one measurable for each $\alpha$ (since $X(t)$ is with probability one continuous). We obtain the following theorem.

THEOREM 3.

$$
\begin{aligned}
\operatorname{Pr}\{\|S\{t \mid X(t)<X(\alpha)\}\|<\beta\}= & \int_{0}^{\beta} f(\alpha, \xi) d \xi, \\
& 0 \leqq \alpha \leqq 1,0 \leqq \beta \leqq 1 .
\end{aligned}
$$

It is readily shown, in fact, that this probability is the limit, for $n \rightarrow \infty$, of $\operatorname{Pr}\left\{\left\|S\left\{t \mid X_{n}(t)<X_{n}(\alpha)\right\}\right\|<\beta\right\}$ for $X_{n}(t)$ defined as above, and Theorem 2 is immediately applicable.

This theorem will give as special cases the distribution of the value 
of $t$ for which $X(t)$ is a maximum $(0 \leqq t \leqq 1)$ and the distribution of the proportion of time for which $X(t)$ is positive $(0 \leqq t \leqq 1)$. We obtain, namely,

$$
\begin{aligned}
\operatorname{Pr}\left\{\sup _{0 \leqq t \leqq 1} X(t)=\sup _{0 \leqq i \leqq \alpha} X(t)\right\} & =\operatorname{Pr}\left\{\int_{0}^{1} \frac{\operatorname{sgn} X(t)+1}{2} d t<\alpha\right\} \\
& =\frac{2}{\pi} \sin ^{-1} \alpha^{1 / 2} .
\end{aligned}
$$

The second expression was proven by $\mathrm{Kac}[3]$ as an illustration of a general technique for evaluating the distribution of certain Wiener functionals.

\section{BIBLIOGRAPHY}

1. E. S. Andersen, On the number of positive sums of random variables, Skandinavisk Aktuarietidskrift vol. 32 (1949) pp. 27-36.

2. $\mathrm{P}$. Erdös and M. Kac, On the number of positive sums of independent random variables, Bull. Amer. Math. Soc. vol. 59 (1946) pp. 401-414.

3. M. Kac, On the distribution of certain Wiener functionals, Trans. Amer. Math. Soc. vol. 65 (1949) pp. 1-13.

UNIVERSITY OF MiCHIGAN 